\title{
Experimental Study of Catheter Ablation Using Ultrasound Energy in Canine and Porcine Hearts
}

\author{
Toyoyuki Oнкuвo, MD, Kaoru OKishige, ${ }^{1}$ MD, \\ Yoshinari Goseki, MD, Tetsu Matsubara, MD, \\ Kazumasa Hiejima, ${ }^{2} \mathrm{MD}$, \\ and Chiharu IBukiyama, MD
}

\begin{abstract}
SUMMARY
We examined the efficacy and safety of ultrasound energy in eliminating the arrhythmogenic substrates of atrial as well as ventricular tissue using a newly developed instrument in both in vivo and in vitro experiments. Ultrasound (US) applicators were tested on 79 lesions created on a beating heart in canine cardiac tissue, and on 64 lesions in porcine heart specimens. US lesions were created by using transducers with frequencies around $5 \sim 10 \mathrm{MHz}$. In the in vivo study, we observed a significant decrease in the amplitude of the electrograms recorded from the tip of the ablation catheter during the US application $(p<0.01)$. In some sites transmural lesions could be created which were well demarcated. Blood coagulum formation was observed on the tip of the ablation catheter on several occasions. In one dog ventricular fibrillation was provoked by the delivery of ultrasound energy to the left ventricle. In the in vitro study, lesion depth increased significantly with a longer duration of energy delivery when the temperature was maintained stable $(p<0.001)$, and the lesion depth increased significantly with higher temperatures of energy delivery when the duration of US application was maintained $(p<0.05)$. In both cases, no significant change in surface area was observed. The maximum depth of the lesion was $10.3 \mathrm{~mm}$. Conclusions: An ultrasound energy system is relatively safe and effective for creating lesions large enough to eliminate arrhythmogenic substrates deep in the ventricular myocardium. Although the US system is free from pop phenomenon, the problem of blood coagulation on the catheter tip remains to be settled. (Jpn Heart J 1998; 39: 399-409)
\end{abstract}

Key words: Ultrasound energy, Catheter ablation, Tachycardia

YATHETER ablation of ventricular tachycardia (VT) associated with
remote myocardial infarction has been reported by several groups to be

From the Second Department of Internal Medicine, Tokyo Medical College, School of Medicine, Tokyo, 'Cardiovascular Division, Yokohama Red Cross Hospital, Yokohama, and 'Department of Health and Hygiene, Tokyo Medical and Dental University, Tokyo, Japan.

Address for correspondence: Kaoru Okishige, MD, Gardiovascular Division, Yokohama Red Cross Hospital,

2-85 Negishi, Naka-Ku, Yokohama 231, Japan.

Received for publication June 4, 1997.

Accepted January 14, 1998. 
successful in only 18 50\% of the survivors even when using direct current shock. ${ }^{1,2)}$ Catheter ablation is successful when the areas from which the VT arises are correctly identified and completely ablated. At present, radiofrequency (RF) energy has been mainly used for the transcatheter ablation of various modalities of tachyarrhythmias. ${ }^{3-6)}$ However, data on catheter ablation of VT by using RF energy is very limited, particularly in patients with structural heart disease presumably because of smaller and shallower lesions. ${ }^{7-10)}$

Ultrasound (US) energy has been used for the treatment of certain types of cancer because of its good penetration through soft tissues at frequencies that control the energy deposition pattern. ${ }^{1-14)}$ US is a form of vibratory energy (more than $18 \mathrm{kHz}$ ) that is propagated as a mechanical wave by the motion of the particles within the medium. ${ }^{15)}$ This wave propagation causes compression and refraction of the particles, and a pressure wave is propagated, associated with the mechanical movement of the particles. An ultrasound field causes particle motion that results in mechanical stress and strain. In an absorbing medium, the ultrasonic energy is continuously absorbed and converted into heat within the medium. ${ }^{16)}$ If the temperature is elevated to some extent and is maintained for a certain period, permanent tissue damage will occur. This thermal effect is similar to that obtained using other heating systems with equal thermal exposure. The objectives of the present study were to examine 1) the feasibility and safety of applying US energy to cardiac tissue both in vivo, and 2) the factors which determine the size of lesions created by US applications in vitro.

\section{Methods}

Ultrasound applicator design, construction and power supply: The US energy generator (The Series $400^{\mathrm{TM}}$ Ultrasound Ablation System, Heart Rhythm Technologies. Inc., Temecula, CA, USA) is designed to deliver temperature controlled (closed loop) US energy to selected sites within the heart. The electrical energy delivered to the US transducer located at the distal end of the catheter is converted into ultrasound energy (Figure 1A). This US energy is then delivered through the ablation catheter (Series $400^{\mathrm{TM}}$ Ultrasound Ablation Catheter, Heart Rhythm Technologies, Inc.). The ablation catheter incorporates a $5 \mathrm{~mm}$ long cylindrical US transducer with three point thermocouples for accurate temperature feedback (Figure 1B). Two electrodes are included for mapping and pacing, one mounted distal to the transducer and the other mounted proximal to the transducer with $5 \mathrm{~mm}$ spacing. The catheter incorporates an integral stiffening mandrel which is used to apply outward tip pressure to ensure wall contact.

In vivo studies: The purpose of the in vivo study was to explore whether US energy would be able to create a lesion in cardiac tissue and to investigate 


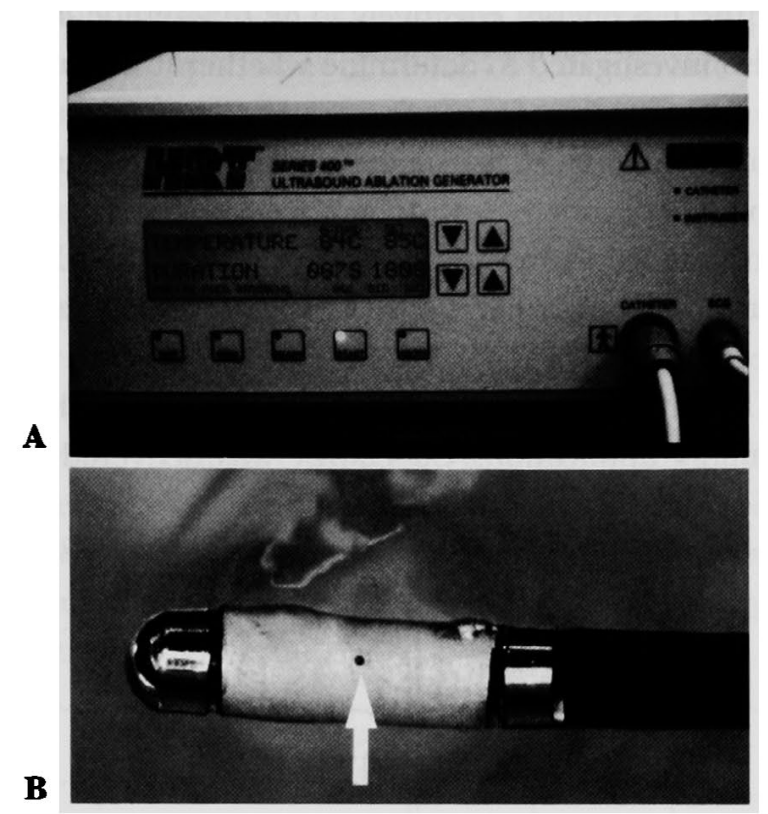

Figure 1. A: Photograph of the ultrasound energy generator: Heart Rhythm Technologies Series $400^{\mathrm{TM}}$ Ultrasound Ablation System. B: Photograph of the ultrasound ablation catheter tip. It has two electrodes separated by the ultrasound transducer of $5 \mathrm{~mm}$ in length. A thermocouple is installed at three points in the catheter tip.

whether the US system would be able to deliver US energy safely to the beating heart without any adverse effects. Seven adult beagles of either sex, weighing $10.9 \mathrm{~kg}$ on average, were used. They were anesthetized with intraveous sodium pentobarbital and intubated and ventilated with room air using a Harvard respirator. The ablation catheter was introduced through the femoral vein or an artery, and then inserted into the right atrium, right ventricle or left ventricle under fluoroscopic guidance. A surface electrocardiogram and the intracardiac uni- and bipolar electrograms obtained from the US ablation catheter were continuously monitored on a recorder (EP Lab, Quinton, Seattle, WA) during the application of US energy. US energy was delivered through the ablation catheter at a preset temperature of $85^{\circ} \mathrm{C}$ for 180 seconds, and the input of the electrical power was automatically adjusted to keep the temperature around the preset value. The US energy was applied to various sites on the endocardium of each cardiac chamber. Significant elevation of the ST segment on the unipolar electrogram, recorded from the proximal as well as distal electrodes which were mounted on both sides of the US transducer, was necessary to determine whether there was adequate stable contact between the US transducer and endocardium 
in order to deliver the US energy effectively. The magnitude of the intracardiac electrogram was also investigated to determine whether it was associated with the size of the lesion.

After the experiment, the beagles were sacrificed and the hearts excised in order to obtain several specimens with which to examine the lesions. All specimens were stained with triphenyl tetrazolium chloride (TTC) to measure the depth of the necrotized tissue created by the US application. The specimens were fixed in $10 \%$ buffered formalin.

In vitro study: To investigate factors which can influence the lesion size created by the US energy application, US energy was delivered by changing the
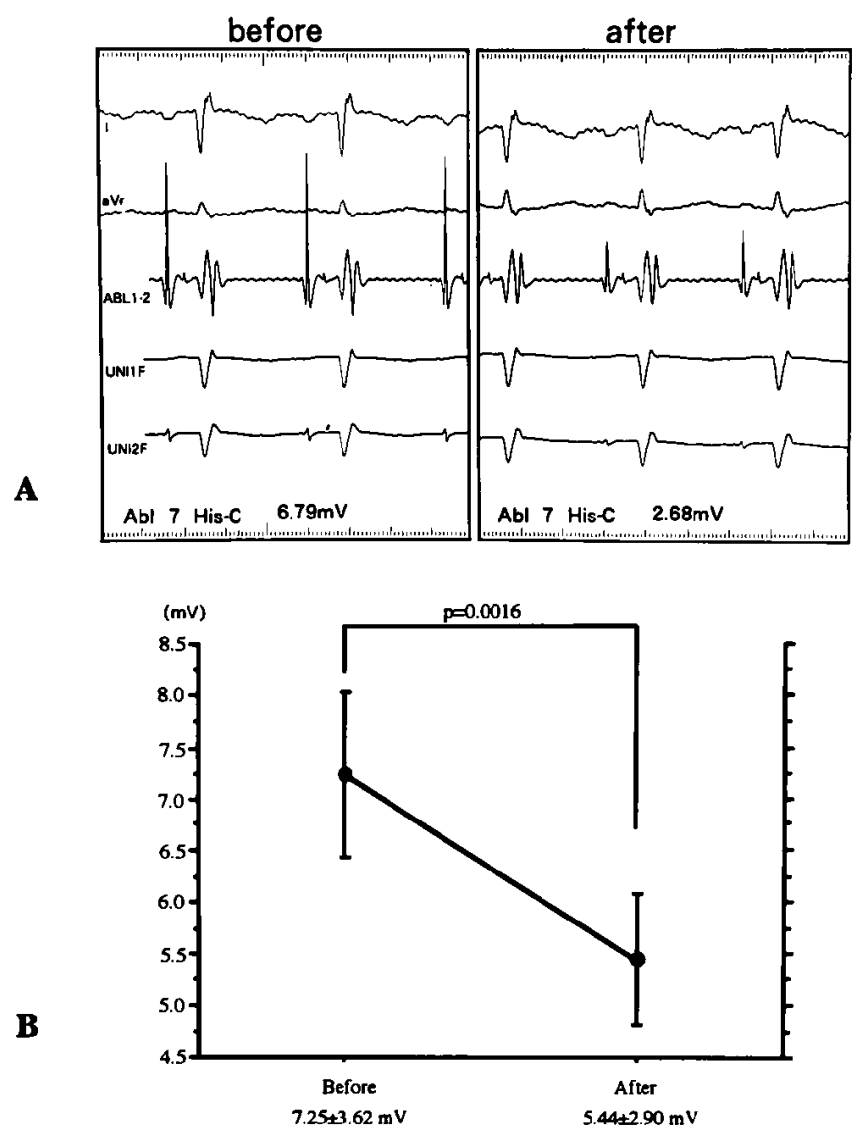

Figure 2. A: Recordings from before (left panel) and after (right panel) the application of the ultrasound energy to the endocardium. There is a significant decrease in the amplitude of the bipolar recording electrogram from 6.79 to $2.69 \mathrm{mV}$.

ABL1-2 = the distal pair of electrodes of the ablation catheter; UNIIF = the distal unipolar electrode; UNI2F = the proxymal unipolar electrode. B: The average change in the amplitude of atrial electrograms before (left panel) and after (right panel) US energy application to the atrial endocardium. The amplitude of the atrial electrograms decreased significantly from $7.25 \pm 3.62$ to $5.44 \pm 2.90 \mathrm{mV}$. 
duration of the US application or changing the preset temperature of the US energy applied to cubic specimens of $20 \mathrm{~mm}$ by $20 \mathrm{~mm}$ size excised from the left ventricle of a porcine heart. The specimens were bathed in warm saline (about $36^{\circ} \mathrm{C}$ ) which was continuously stirred during the procedure. The lesion size was examined 1) according to the change in the exposure duration of $60,120,180$, 240 and 300 seconds at a constant temperature of $85^{\circ} \mathrm{C}$, and 2) according to the change in the temperature at 70,80 and $90^{\circ} \mathrm{C}$ with the same application interval of $180 \mathrm{sec}$. After each application of US energy, each specimen was cut in the center of the block to measure the depth and area.

Statistical analysis: Data are expressed as mean \pm standard deviation. Statistical analyses were performed using the one-way factorial ANOVA and Fisher's Protected Least Siginificant Difference (Fisher's PLSD) methods for the in vitro study, and the paired student's $t$ test for the in vivo study. A value of $p<0.05$ was considered statistically significant.

\section{Results}

In vivo study: A total of 79 lesions were created: 9 in the right atrium, 31 along the tricuspid annulus (including the compact AV node area), 13 in the right ventricular free wall, and 26 in the left ventricle. The amplitude of the atrial electrograms recorded from the bipolar electrodes placed on the tip of the ablation catheter positioned in the right atrium decreased significantly with the application of US energy (Figure 2A). The average of the amplitude of the atrial electrograms changed from $7.25 \pm 3.62$ to $5.44 \pm 2.90 \mathrm{mV}(p=0.002)$ in a gradual fashion (Figure 2B). We failed to examine the change in the ventricular electrograms because of saturation of the electrograms during the procedure. AV nodal conduction could not be modified by US energy application to the anterosuperior portion of Koch's triangle, which corresponds to the compact AV node, despite delivery of the highest energy level.

Sonication produced conical shaped lesions of various sizes in the endocardium, all of which were very well demarcated. After TTC staining, transmural lesions in the right atrium were observed, and deep lesions were also observed in the ventricular tissue without any coagulum formation occurring (Figure 3). In this case, the surface of the lesion had a crater formation due to the high catheter pressure against the endocardium. Arterial blood pressure did not change significantly in any of the cases, however, ventricular fibrillation was provoked with the initial application of US energy to the left ventricle in one canine. In a few cases, blood coagulum was observed on the surface of the US transducer, which prevented an efficient increase in temperature during the US energy application. Pop phenomenon was not induced in any of the cases. 


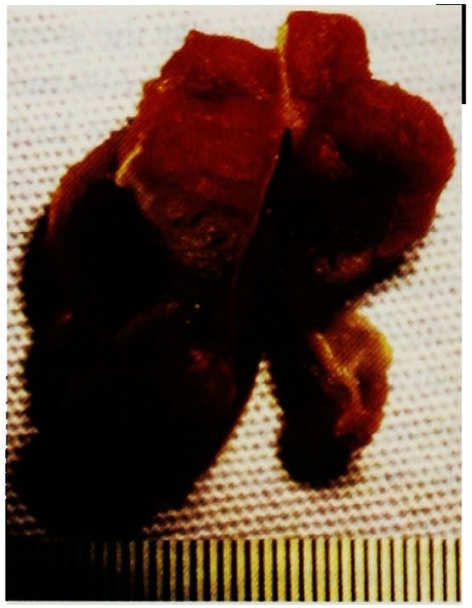

Figure 3. This specimen was excised from the left ventricle of the canine heart. The cross section shows the lesion created by the ultrasound energy application at a temperature of $85^{\circ} \mathrm{C}$ for 180 seconds. The lesion is well demarcated in the left ventricular wall. In addition, no coagulum formation was observed.

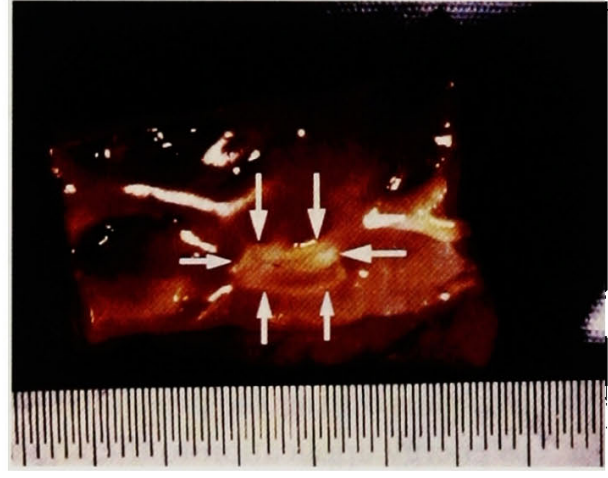

A

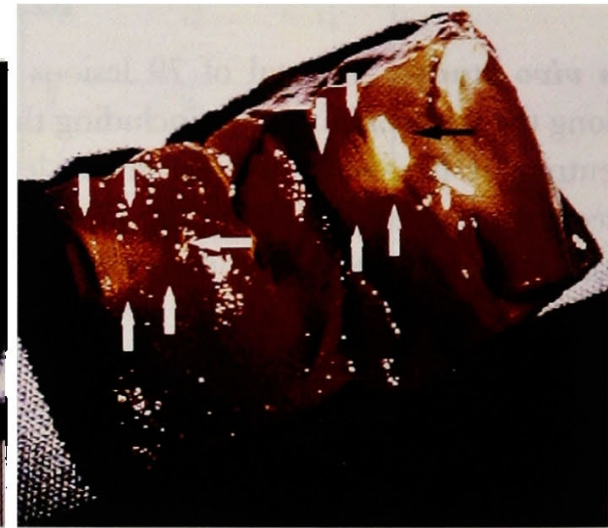

B

Figure 4. This photograph shows the surface area $(\mathbf{A})$ and the depth $(\mathbf{B})$ of the lesion created by US energy application in the porcine heart specimen. The morphology of the surface area of the lesion is rectangular. In this case, the US energy was applied at a temperature of $80^{\circ} \mathrm{C}$ for 180 seconds.

In vitro study: A total of 69 lesions were made in the left ventricle of the porcine heart specimens. Lesions could be created by application of the US energy for at least approximately 15 seconds. The endocardial surface of the lesions was rectangular and well demarcated from the surrounding tissue (Figure 4). In this case, the US energy was applied at a temperature of $80^{\circ} \mathrm{C}$ for 180 seconds. Lesion depth increased significantly as the duration of the US delivery was increased for 60 to 300 seconds at the same temperature. When the temperature was set at $85^{\circ} \mathrm{C}$, the average depth of the lesion was $2.8 \pm 0.6,4.8 \pm 1.0$, 

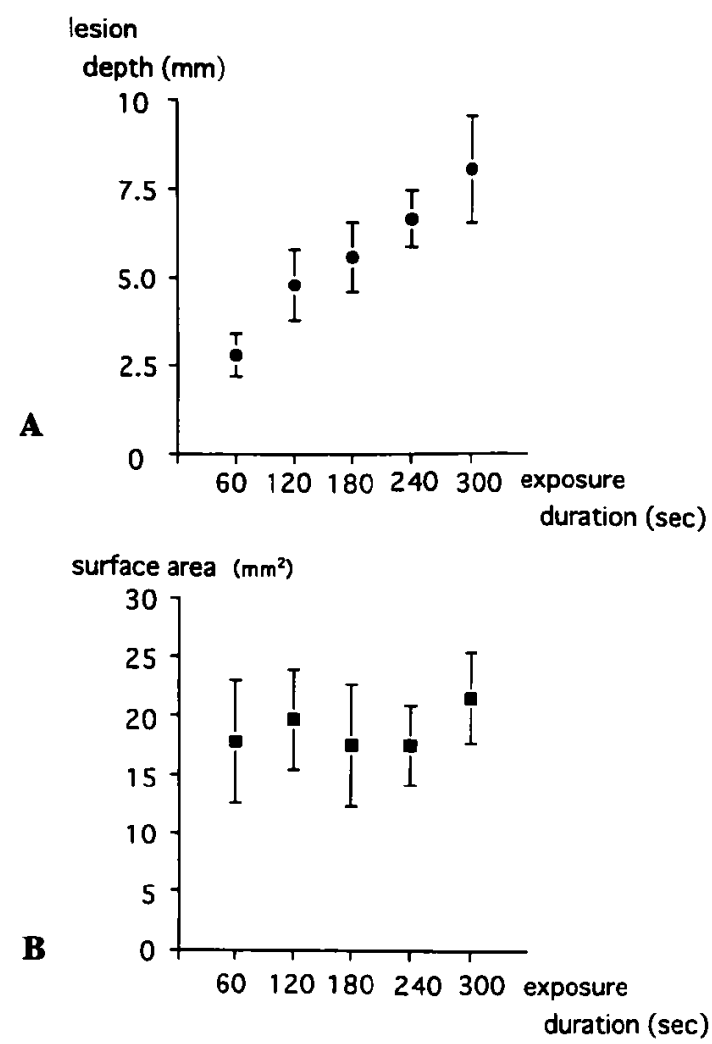

Figure 5. A: The lesion depth increased significantly as the duration of US energy application was increased when the preset temperature of $85^{\circ} \mathrm{C}$ was maintained $(p<0.001, n=9)$. B: There were no significant differences in the changes in surface area among the various durations of US energy applied $(p=0.18)$.

$5.6 \pm 1.0,6.7 \pm 0.8$ and $8.1 \pm 1.5 \mathrm{~mm}$ at a delivery duration of $60,120,180,240$, and 300 seconds, respectively $(p<0.001, n=9$ ) (Figure 5A). Using the post hoc test, statistical significance was recognized in every chosen pair of values among the results, except for the comparison between 180 and 240 seconds. However, the surface area of the lesion did not show any significant differences according to the duration of US application $(p=0.18)$. The average surface areas of the lesions were $17.8 \pm 5.3,19.7 \pm 4.3,17.5 \pm 5.3,17.5 \pm 3.4$, and $21.5 \pm 3.9 \mathrm{~mm}^{2}$ at delivery durations of 60, 120,180, 240, and 300 seconds, respectively (Figure 5B). In contrast, when the total duration of the US application was set for 180 seconds, the average depth of the lesion increased significantly according to the temperature $(\phi<0.05)$. The average depth of the lesion was $4.0 \pm 1.3,6.4 \pm 2.2$, and $8.0 \pm 1.5 \mathrm{~mm}$ at temperatures of 70,80 , and $90^{\circ} \mathrm{C}$, respectively $(n=8)$ (Figure $6 \mathrm{~A})$. Using the post hoc test, statistical significance was observed with the comparison of the average depth between that at 70 and $90^{\circ} \mathrm{C}$. However, the surface area did not show the significant differences according to temperature $(p=0.24)$. 

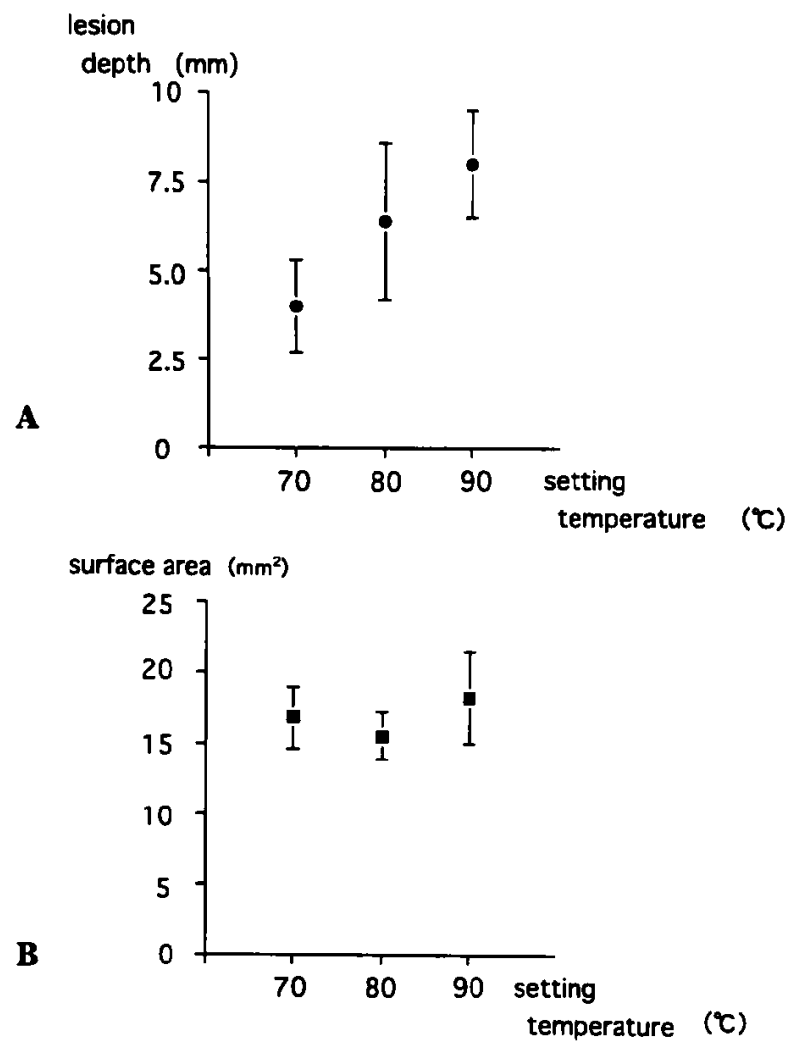

Figure 6. A: The lesion depth increased significantly as the preset temperature was increased from 70 to $90^{\circ} \mathrm{C}(\rho<0.05, n=8)$. B: There were no significant differences in the changes in the surface area among the preset temperatures $(p=0.24)$.

The average surface areas of the lesions were $16.8 \pm 2.2,15.5 \pm 1.7$, and $18.2 \pm 3.2 \mathrm{~mm}^{2}$ at temperatures of 70,80 , and $90^{\circ} \mathrm{C}$, respectively (Figure 6B). The maximum surface area of the lesion was $29.4 \mathrm{~mm}^{2}$, and the maximum depth of the lesion in the left ventricle was $10.3 \mathrm{~mm}$, which was created by an exposure duration of 300 seconds at a temperature of $85^{\circ} \mathrm{C}$. Two episodes of an abnormal increase in the tip temperature occurred, however, the cause has not been determined.

\section{Discussion}

The present experiment provides in vivo as well as in vitro evidence indicating that US energy can produce sufficient lesions which should be suitable for intracardiac ablation of arrhythmias. US has not been previously investigated for use with intracardiac ablation because of the assumption that sufficient energy could not be delivered through a small size transducer to be used for this purpose. ${ }^{16)}$ 
The present US ablation system was constructed to deliver a higher energy output resulting in higher efficiency. With radiofrequency energy the average lesion depth is usually $3 \sim 5 \mathrm{~mm} .{ }^{17)}$ This lesion depth is sufficient to eliminate most accessory pathways and atrial tachycardias, including atrial flutter and AV nodal reentrant tachycardia. However, ventricular tachycardias associated with remote myocardial infarction may require greater penetration in the myocardial tissue for successful ablation. ${ }^{18-20)}$ The results of the present study demonstrate that deeper and larger lesions sufficient for complete elimination of the arrhythmogenic foci of various kinds of tachyarrhythmias which have been intractable to the current radiofrequency ablation system can be created with this US ablation system. ${ }^{21)}$

US energy ablation has several peculiar characteristics different from other kinds of energy sources. First, the configuration of the surface area of the lesion created by US application is rectangular rather than hemispheric, which enables the US waves to penetrate deeper into the myocardium. Second, US energy causes heat in the myocardium by mechanical compression and refraction waves, therefore, US energy is free from vibratory interference with surface ECG as well as intracardiac recordings. This enables us to monitor the changes in intracardiac unipolar and bipolar electrograms during US energy application. The effects of US energy on the endocardium can be monitored as a decrease in the amplitude of the electrograms recorded through the tip electrodes of the ablation catheter. Therefore, we are able to detect whether the US energy is effectively delivered to the endocardium. Moreover, elevation of the ST segment of the electrograms recorded from the unipolar electrodes placed on the tip of the ablation catheter can provide information regarding the status of contact of the US transducer against the endocardial surface, which is indispensable for effective delivery of US energy.

Although this US ablation system is very promising, this technology does not seem to be suitable for ablation of accessory pathways located on either side of the cardiac chamber. The problem is the difficulty encountered in the manipulation of the ablation catheter in order to position the US transducer completely against the endocardial wall. We think that the present US ablation system can successfully be applied to ventricular tachycardias whose focus lies buried deep in the myocardium, to AV nodal modification, and to common atrial flutter. US energy is unable to penetrate dense fibrous tissue (personal communication with Dr. Frank I. Marcus), and therefore, the US system may not be suitable for ablation of the $\mathrm{AV}$ junction.

US ablation catheters seem to carry the risk of causing blood coagulum on the US transducer, and therefore, anticoagulation with intravenous heparin is recommended during the US ablation procedure. Since no clue has been found 
to predict the onset of blood coagulum formation with this system, much attention should be paid to monitoring each parameter to determine the status of US delivery, including temperature and unipolar or bipolar electrograms obtained from the ablation catheter tip. In contrast, one of the advantages of US energy is that it is free from pop phenomenon because it creates the lesion by vibration energy.

A single episode of ventricular fibrillation (VF) was observed in one case, and the cause of this phenomenon is still unknown. The manner in which the US energy was applied to the canine hearts was identical in all cases, and VF was provoked on only one occasion. Therefore, it was almost impossible to determine whether or not the US ablation system has some proarrhythmic effects.

Conclusion: This US energy ablation system can be applied to the myocardium with a more effective and deeper penetration into the myocardium than with radiofrequency energy. Higher temperatures and longer durations of the US application are able to create larger lesions. There is a risk of blood coagulum formation but no risk of pop phenomenon during US delivery in the heart.

\section{REFERENCES}

1. Borggrefe M, Breithard G, Podczeck A, et al. Catheter ablation of ventricular tachycardia using defibrillator pulses:electrophysiological findings and long-term results. Eur Heart J 1989; 10: 591-601.

2. Garan H, Kuchar D, Freeman C, et al. Early assessment of the effect of mao-guided transcatheter intracardiac electrical shock on sustained ventricular tachycardia secondary to coronary artery disease. Am J Cardiol 1988; 61: 1018-23.

3. Jackman WM, Wang X, Friday KJ, et al. Catheter ablation of accessory atrioventricular pathways (Wolff-Parkinson-White Syndrome) by radiofrequency current. N Engl J Med 1991; 324: 1605-11.

4. Kay GN, Epstein AE, Dailey SM, et al. Selective radiofrequency ablation of the slow pathway for the treatment of atrioventricular nodal reentrant tachycardia. Circulation 1992; 85: 1675-88.

5. Tracy CM, Swartz JF, Fletcher RD, et al. Radiofrequency catheter ablation of ectopic atrial tachycardia using paced activation sequence mapping. J Am Coll Cardiol 1993; 21: 910-7.

6. Katritsis D, Heald S, Ahsan A, et al. Catheter ablation for successful management of left posterior fascicular tachycardia: an approach guided by recording of fascicular potentials. Heart 1996; 75: 3848.

7. Morady F, Harvey M, Kalbfleisch SJ, et al. Radiofrequency catheter ablation of ventricular tachycardia in patients with coronary artery disease. Circulation 1993; 87: 363-72.

8. Stevenson WG, Khan $\mathrm{H}$, Sager $\mathrm{P}$, et al. Identification of reentry circuit sites during catheter mapping and radiofrequency ablation of VT late after myocardial infarction. Circulation 1993; 88: 1647-70.

9. Kim YH, Sosa-Suarez G, Trouton TG, et al. Treatment of ventricular tachycardia by transcatheter radiofrequency ablation in patients with ischemic heart disease. Circulation 1994; 89: 1094-102.

10. Gonska BD, Cao K, Schaumann A, et al. Catheter ablation of ventricular tachycardia in 136 patients with coronary artery disease: results and long-term follow-up. J Am Coll Cardiol 1994; 24: 1506-14.

11. Hynynen K. Methods of external hyperthermic heating in clinical thermology. In: Gautherie editor. Subseries, Thermotherapy. Heidelberg: Springer-Verlag. 1990: 61-115.

12. Corry PM, Barlogie B, Tilchen EJ. Ultrasound induced hyperthermia for the treatment of human superficial tumors. Int J Radiat. Oncol Biol Phys 1982; 8: 1225-9.

13. Hynynen K, Roemer R, Anhalt D. A scanned, focused, multiple transducer ultrasonic system for localized hyperthermia treatments. Int J Hyperthermia, 1987; 3: 21-35. 
14. Marmor JB, Pounds D, Postic TB. Treatment of superficial human neoplasms by local hyperthermia induced by ultrasound. Cancer 1979; 43: 188-97.

15. Stewart HF. Ultrasonic measurement techniques and equipment output levels. In: Repacholi $\mathbf{M H}$, Benwell DA, editors. Essentials of Medical Ultrasound. Clifton NJ: The Humana Inc. 1982: 77-1 16.

16. He DS, Zimmer JE, Hynynen K, et al. Application of ultrasound energy for intracardiac ablation of arrhythmias. Eur Heart J 1995; 16: 961-6.

17. Haines DE. Current and future modalities of catheter ablation for the treatment of cardiac arrhythmias. J Invas Cardiol 1992; 4: 291-9.

18. Huang SS, Graham AR, Wharton K. Radiofrequency catheter ablation of the left and right ventricles: anatomic and electrophysiologic observations. PACE 1988; 11: 449-57.

19. Willems S, Borggrefe M, Shenasa M, et al. Radiofrequency catheter ablation of ventricular tachycardia following implantation of an automatic cardioverter defibrillator. Pacing Clin Electrophysiol 1993; 16: 1684-92.

20. Gursoy S, Chiladakis I, Kuck KH. First lessons from radiofrequency catheter ablation in patients with ventricular tachycardia. Pacing Clin Electrophysiol 1993; 16: 687-91.

21. Zimmer JE, Hynynen K, Marcus FI. The feasibility of using ultrasound for cardiac ablation. IEEE Ultrasound Symp; 1993; 1203-6. 